\title{
Experimental and molecular dynamic simulation study of perfluorooctane sulfonate adsorption on soil and sediment components
}

\author{
Ruiming Zhang, Wei Yan, Chuanyong Jing* \\ State Key Laboratory of Environmental Chemistry and Ecotoxicology, Research Center for Eco-Environmental Sciences, \\ Chinese Academy of Sciences, Beijing 100085, China. E-mail: zhangruim07@163.com
}

\section{A R T I C L E I N F O}

Article history:

Received 30 October 2014

Revised 31 October 2014

Accepted 13 November 2014

Available online 8 January 2015

Keywords:

Humic/fulvic acid

Humin/kerogen

PFOS

Adsorption

Molecular dynamics

\begin{abstract}
A B S T R A C T
Soil and sediment play a crucial role in the fate and transport of perfluorooctane sulfonate (PFOS) in the environment. However, the molecular mechanisms of major soil/sediment components on PFOS adsorption remain unclear. This study experimentally isolated three major components in soil/sediment: humin/kerogen, humic/fulvic acid (HA/FA), and inorganic component after removing organics, and explored their contributions to PFOS adsorption using batch adsorption experiments and molecular dynamic simulations. The results suggest that the humin/kerogen component dominated the PFOS adsorption due to its aliphatic features where hydrophobic effect and phase transfer are the primary adsorption mechanism. Compared with the humin/kerogen, the HA/FA component contributed less to the PFOS adsorption because of its hydrophilic and polar characteristics. The electrostatic repulsion between the polar groups of HA/FA and PFOS anions was attributable to the reduced PFOS adsorption. When the soil organic matter was extracted, the inorganic component also plays a non-negligible role because PFOS molecules might form surface complexes on $\mathrm{SiO}_{2}$ surface. The findings obtained in this study illustrate the contribution of organic matters in soils and sediments to PFOS adsorption and provided new perspective to understanding the adsorption process of PFOS on micro-interface in the environment.

(c) 2014 The Research Center for Eco-Environmental Sciences, Chinese Academy of Sciences.
\end{abstract}

Published by Elsevier B.V.

\section{Introduction}

Perfluorooctane sulfonate (PFOS), consisting of a sulfonate group and 8-carbon C-F tails (Appendix A Fig. S1), has been identified as an emerging contaminant of global concern (Nakayama et al., 2010; Zhang et al., 2013). Worldwide distribution and contamination of PFOS pose great threat not only to the environment, but also to the animal and human health (Beach et al., 2006; Betts, 2008; Nelson et al., 2010). PFOS can bind to peroxisome proliferator-activated receptors which are associated with carcinogenesis, affect growth and development, and even disrupt the hormone and immune systems (Betts, 2007). Hence, a long-term and high exposure to PFOS can lead to severe symptoms such as endocrine disruption and cancer (Betts, 2007), physical development delay (Gump et al., 2011), and neonatal mortality (Kannan et al., 2010; Luebker et al., 2005).

The fate and transport of PFOS in the environment greatly depend on its adsorption on soil and sediment (Chen et al., 2012;

\footnotetext{
* Corresponding author. E-mail: cyjing@rcees.ac.cn (Chuanyong Jing).
} 
Higgins and Luthy, 2006; Johnson et al., 2007; Ochoa-Herrera and Sierra-Alvarez, 2008). Soil and sediment are complicated matrices and generally composed of multiple complex components, which could be operationally defined as humin/kerogen, humic/fulvic acid (HA/FA), and inorganic component after chemical treatment (Mikutta et al., 2005). These components may impact PFOS adsorption with different mechanisms. However, soil and sediment are usually deemed as a single entity to investigate PFOS adsorption using kinetics and isotherm experiments (Higgins and Luthy, 2006, 2007; You et al., 2010). The lack of molecular-level knowledge of PFOS interaction with different soil/sediment components may limit our understanding and prediction of the PFOS behavior in the environment.

Molecular dynamic (MD) simulation can provide molecular level morphological and structural details on the solid-aqueous interface and has been used to study the surface adsorption and transport of pollutants. For example, MD simulation was successfully applied to study the mechanism of benzene uptake in the interlayer of montmorillonite with alkyl-methylammonium as organic substances (Zhao and Burns, 2012, 2013). Their MD results well explained the molecular mechanisms of experimental observations that the uptake capacity of organic pollutants increased with the increasing total organic content in organoclays. In our study, MD simulation was applied, as a complimentary technique to experimental observations, to investigate the role of soil/sediment components on PFOS adsorption.

The objective of our work was to explore the molecular mechanisms of PFOS adsorption on soil/sediment components. The three major components, i.e., humin/kerogen, HA/FA, and inorganic component, were isolated using extraction methods and their effects were studied with PFOS adsorption experiments. MD simulations were employed to study the interaction between PFOS and surfaces at the molecular level. Our experimental and model simulation results should improve the understanding of the solid-aqueous partition mechanisms of PFOS on environmental matrices.

\section{Materials and methods}

\subsection{Materials}

Five soil samples and one sediment sample with a wide range of total organic carbon (TOC) content (1.0\%-8.3\%) were collected. The soil samples were collected on the surface horizon $(0-20 \mathrm{~cm})$ from Liaoning Province (SY, LN), Heilongiiang Province (HL) and Beijing (BJ1, BJ2) of China, respectively. The sediment (SE) was sampled from Qinghe River in Beijing. The samples were air-dried, sieved $(<2 \mathrm{~mm})$ and stored at room temperature. The sample properties are given in Appendix A Table S1. Two treatment methods with $\mathrm{NaOH}$ and $\mathrm{H}_{2} \mathrm{O}_{2}$ were carried on to remove $\mathrm{HA} / \mathrm{FA}$ and organic substances including HA/FA and humin/kerogen, respectively (Liu et al., 2008; Shi et al., 2010).

For the $\mathrm{NaOH}$ treatment method, HA/FA was removed, and the remaining fractions were humin/kerogen substances and inorganic component. The soil/sediment and $0.5 \mathrm{~mol} / \mathrm{L} \mathrm{NaOH}$ were mixed at a solid/solution ratio of 1:50 for at least 8 times until the solution was colorless, and each time for $6 \mathrm{hr}$ (Shi et al., 2010).

For the $\mathrm{H}_{2} \mathrm{O}_{2}$ treatment method, HA/FA and humin/ kerogen substances were removed, and the remaining fractions in residual were mainly inorganic component such as $\mathrm{SiO}_{2}$ and metal oxides (Mikutta et al., 2005). Samples were mixed with $10 \% \mathrm{H}_{2} \mathrm{O}_{2}$ solution at a solid/solution ratio of 1:50 for at least 3 times, each time for $6 \mathrm{hr}$ (Mikutta et al., 2005). After treatment, the TOC in the treated sample was measured, and the results are shown in Appendix A Table S1. Moreover, the TOC was consisted of $35.2 \%-75.9 \%$ HA/FA and $24.1 \%-64.8 \%$ humin/kerogen substances as shown in Appendix A Fig. S2.

\subsection{Batch sorption}

In kinetics experiments, an initial concentration of $200 \mu \mathrm{g} / \mathrm{L}$ PFOS solution in $0.01 \mathrm{~mol} / \mathrm{L} \mathrm{NaCl}$ was added to a $100 \mathrm{~mL}$ polypropylene bottle, adjusting with $\mathrm{HCl}$ and $\mathrm{NaOH}$ to keep the $\mathrm{pH}$ at 7.0 \pm 0.1 . At designated time intervals in a period of 7 days, a $1.5 \mathrm{~mL}$ sample was collected and centrifuged at 10,000 r/min for $20 \mathrm{~min}$, and then PFOS in the supernatant was analyzed.

Adsorption isotherm experiments were carried out in $15 \mathrm{~mL}$ corning polypropylene tubes. Duplicate suspension samples contain $15 \mathrm{~g} / \mathrm{L}$ of the raw/treated soil/sediment samples and 10-500 $\mu \mathrm{g} / \mathrm{L}$ PFOS solution under the same experimental conditions to kinetics. After mixing on a rotator for 7 days, the suspension was centrifuged at 10,000 r/min for $20 \mathrm{~min}$. Then, the supernatant was collected for PFOS analysis.

\subsection{PFOS analysis}

Dissolved PFOS concentrations were measured using an Agilent 1290 infinity liquid chromatography (Agilent Technologies, Palo Alto, CA, USA) coupled with an Agilent 6540 Ultra High Definition Q-TOF mass spectroscopy (Agilent Technologies, Santa Clara, CA, USA). Chromatographic separation was performed on an XBridge $^{\text {TM }}$ C-18 column $(2.1 \times 100 \mathrm{~mm}, 3.5 \mu \mathrm{m}$; Waters Corp., Dublin, Ireland) with an injection of $10 \mu \mathrm{L}$ samples. The mobile phase consisted of $10 \mathrm{mmol} / \mathrm{L}$ ammonium acetate and acetonitrile at a molar ratio of 30:70. The operation parameters were as follows: capillary voltage $3500 \mathrm{~V}$ in negative; nebulizer pressure 35 psig; drying gas $10 \mathrm{~L} / \mathrm{min}$; gas temperature $350{ }^{\circ} \mathrm{C}$; sheath gas flow $11 \mathrm{~L} / \mathrm{min}$; sheath gas temperature $250^{\circ} \mathrm{C}$; nozzle voltage $1000 \mathrm{~V}$ in negative ion mode; and the fragmentor voltage $60 \mathrm{~V}$. The data was processed with MassHunter software (Agilent Technologies). The detection and quantification limit of PFOS were $0.3 \mu \mathrm{g} / \mathrm{L}$ and $1.0 \mu \mathrm{g} / \mathrm{L}$, respectively. The blanks without adsorbent showed that the total PFOS loss on the centrifuge tube wall was less than $3.6 \%$ of the initial concentration. Therefore, the uptake of PFOS by solids was calculated using the mass balance.

\subsection{Model rationale}

In $\mathrm{MD}$ simulation, simple organic molecules are often used to stand for complicated natural organic matter. For example, gallic acid, protocatechuic acid, 4-hydroxybenzoic acid (Giannakopoulos et al., 2006),tetramer undecanoid acid (Aquino et al., 2011), and benzoic acid (Sun et al., 2013) have been applied to simulate humic acid and dissolved organic matter.

The organic substances in soil/sediment are consisted of various components with different structures and functional groups. In these components, HA/FA and humin/kerogen are two general constituents. HA/FA contains much polar organic carbon with polar functional groups, whereas humin/kerogen are enriched in condensed structures and lead to higher sorption affinity to nonpolar solutes (Kang and Xing, 2005; Luo et al., 2008; Wang et al., 2011). Meanwhile, humin/kerogen are more aliphatic and less polar than HA/FA because humin/ kerogen contains mainly aliphatic and aromatic carbon with 
less polar functional groups such as carboxyl (Kang and Xing, 2005). Based on these discrepancies in structural and polar properties, hexane and butylbenzene were chosen to represent humin/kerogen, and butylbenzoic acid with - $\mathrm{COOH}$ group was used to emblematize $\mathrm{HA} / \mathrm{FA}$. Silicate $\left(\mathrm{SiO}_{2}\right)$ was used as a model clay mineral because it is the primary and stable composition in inorganic component (Mikutta et al., 2005). The detailed building processes of solid-aqueous layer models are provided in the Appendix A Supplementary data.

\subsection{MD simulation}

All molecular dynamics simulations were carried out using the Forcite module with the COMPASS force field (Sun, 1998). The water was calculated by the simple point charge model (Zhao et al., 2010). All initial solid/water interface configurations were minimized using smart minimization before running the dynamics. This stepwise scheme entails steepest-descent switching to conjugated gradient method as the energy derivatives decrease in order to accelerate the computation (Zhao et al., 2010). Equilibration and subsequent simulations were conducted in the canonical (NVT) ensemble. The system temperature was maintained at $298 \mathrm{~K}$ using the Andersen thermostat with an integration time step of $1.0 \mathrm{fs}$ (He et al., 2013). The equations of motion were integrated using the velocity Verlet algorithm (Verlet, 1967). Initial velocities were randomly assigned according to the Boltzmann distribution ( $\mathrm{He}$ et al., 2013), then used as the current velocity. The electrostatic interactions were calculated with the Ewald summation method (Plimpton, 1995), and the van der Waals interactions were handled by atom-based summation method with a cutoff distance of $9.5 \AA$ (Zhao et al., 2010). Each simulation for all studied systems was preceded by a pre-equilibration period of 100 ps and followed with 2000 ps to reach equilibrium.

\section{Results and discussion}

\subsection{Batch adsorption experiments}

In the kinetics experiments, soil and sediment treated with different methods were used to represent various soil components as adsorbent for PFOS. As shown in Fig. 1, a fast uptake of PFOS from solution to solid surface was observed within the first 2 days, and the equilibrium was reached in all systems within 7 days. The kinetics could be well simulated using the pseudo second-order model, and the related parameters are listed in Appendix A Table S2.

The adsorption isotherms of PFOS are illustrated in Fig. 2 and exhibit nonlinear characters. The isotherms conform to Langmuir models for all samples. The PFOS adsorption capacity ranged 15.1-32.3 $\mu \mathrm{g} / \mathrm{g}$ on raw soils and sediments (Appendix A Table S3). This adsorption capacity was in consistent with previous reported values of $13.1-66.8 \mu \mathrm{g} / \mathrm{g}$ on sediments (Johnson et al., 2007; You et al., 2010).

The adsorption capacity of PFOS was reduced with the decrease in TOC of treated soil/sediment samples (Fig. 2 and Appendix A Table S3), suggesting that organic substances play a key role in PFOS adsorption. Furthermore, humin/ kerogen is the dominant organic substance for PFOS adsorption on soil/sediment. For example, PFOS adsorption was reduced by $6.2 \%-16.4 \%$ and $29.9 \%-55.1 \%$, respectively, due to the removal of HA/FA and humin/kerogen (Fig. 3). Humin/kerogen showed a higher contribution to PFOS adsorption than HA/FA because the condensed and cross-linked structures in humin/kerogen enable its higher surface hydrophobicity and larger van der Waals forces than HA/FA (Han et al., 2013; Shi et al., 2010).

Inorganic minerals, mainly silicates, were the primary constituents in soil/sediment, and attributed to $35.1 \%-53.6 \%$ PFOS adsorption (Fig. 3). Similarly, high (46\%-66\%) contributions of inorganic soil component to 1,3,5-trinitrobenzene adsorption were reported (Shi et al., 2010). In natural conditions, however, most inorganic minerals are coated with organic substances, and therefore the sorption sites on inorganic component might not be fully available (Shi et al., 2010). Thus, the contribution of inorganic component to PFOS adsorption might be overestimated in batch experimental study. Hence, the high contributions of humin/kerogen (29.9\%-55.1\%, Fig. 3) and HA/FA (6.2\%-16.4\%) may even be underestimated.

HA/FA, humin/kerogen, and inorganic component play a significant role in PFOS adsorption. However, their molecularlevel interactions are still unclear. Thus, the mechanisms of PFOS uptake on different soil/sediment components were explored using MD simulation.

\subsection{Equilibrium structures and density profiles by MD simulation}

The effect of different components on PFOS adsorption could be evidenced by adsorption structures and density profiles, which provide information on molecular force, dynamics, and concentration distribution. The 8-PFOS loading systems were established (Appendix A Fig. S3) and their snapshots at equilibrium are shown in Fig. 4 . The density profiles of PFOS along the $z$ direction (normal to the basal surfaces) are illustrated in Fig. 5.

For $\mathrm{SiO}_{2}$ surface, six out of 8 PFOS molecules were adsorbed on the surface, and their $\mathrm{C}-\mathrm{F}$ tails and $\mathrm{SO}_{3}$ groups could contact with the surface (Fig. 4a). The PFOS density profile exhibited in Fig. 5 showed a strong peak near zero (3.7 $\AA$ ) and a weak peak at $21 \AA$ above the $\mathrm{SiO}_{2}$ surface. The strong peak suggested that PFOS was adsorbed on $\mathrm{SiO}_{2}$, consistent with batch experimental results that a range of $35.1 \%-53.6 \%$ PFOS was adsorbed on inorganic component. Furthermore, the structural configurations indicated that the adsorbed PFOS molecules interacted with $\mathrm{SiO}_{2}$ surfaces by both $\mathrm{C}-\mathrm{F}$ tails and $\mathrm{SO}_{3}$ groups. This interaction may be due to hydrophobic and specific chemical adsorption for PFOS on $\mathrm{SiO}_{2}$ (Tang et al., 2010).

For hexane modified surface, the adsorbed PFOS molecules were adjacently well-distributed on hexane modified surface (Fig. 4b), also exhibiting a strong peak at $3.7 \AA$ and a remote peak near $47 \AA$ (Fig. 5). The uniform distribution on hexane surface could be explained by the hydrophobic effect and the phase transfer (or absorbance) between water phase and organic phase (Hu et al., 2013; Zhao and Burns, 2012). Indeed, the aliphatic part of organic materials could play as organic phase (Higgins and Luthy, 2007). The butylbenzene modified surface also attracted PFOS close to the surface, forming a well distributed layer (Fig. 4c). It was similar to the hexane 


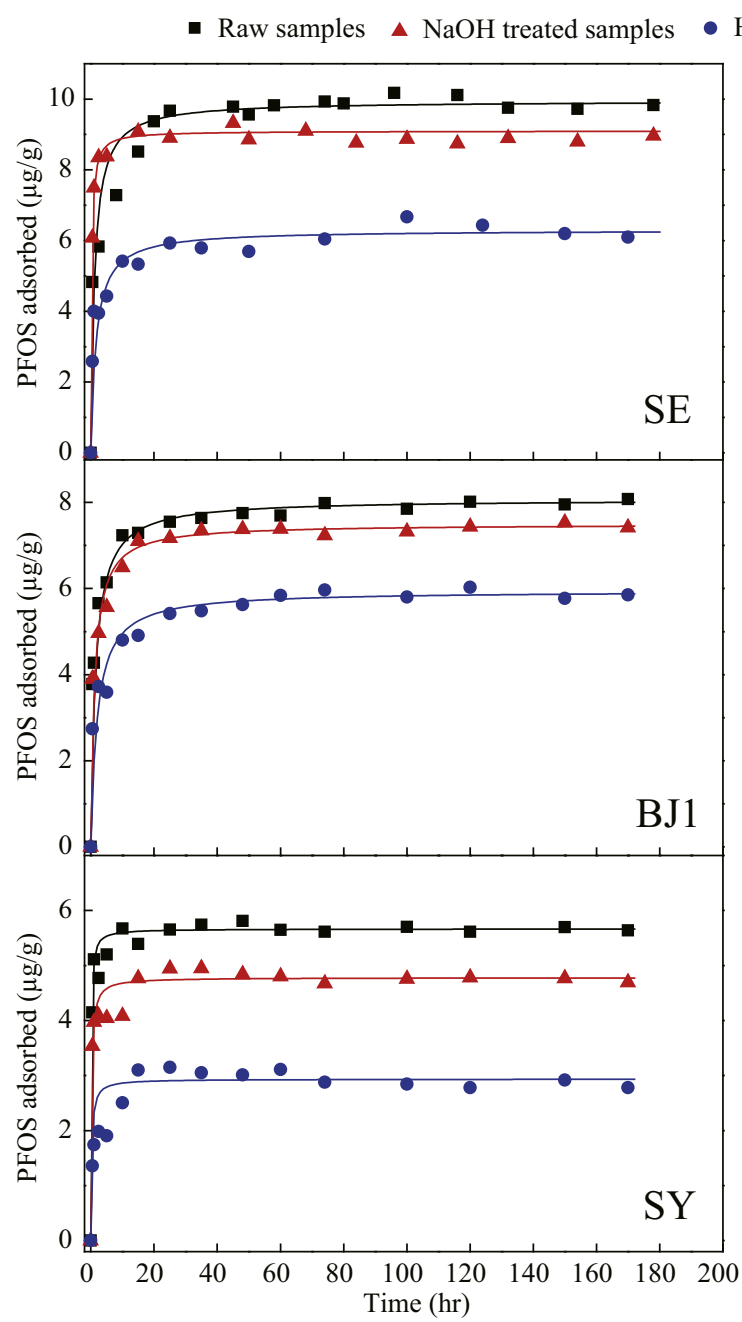

$\mathrm{H}_{2} \mathrm{O}_{2}$ treated samples — Pseudo second-order model

Fig. 1 - Adsorption kinetics of perfluorooctane sulfonate (PFOS) on different soil/sediment samples.

modified surface and a strongest peak near $1.8 \AA$ was observed in Fig. 5. This phenomenon was consistent with our experimental data that humin/kerogen was as the main component contributed to PFOS adsorption (Fig. 3), suggesting the high affinity of PFOS to aliphatic and nonpolar surface.

In contrast, for butylbenzoic acid modified surface, almost no peak near the surface was observed, but a broad peak at 5-30 ̊ (Fig. 5). The MD results show that only one PFOS molecule was inserted into the organic layer of butylbenzoic acid modified surface (Fig. 4d); other PFOS molecules were aggregated to form micelle above the surface. This distribution indicated that PFOS is inclined to aggregate in bulk solution rather than adsorb on surface. These phenomena may be attributed to the high hydrophilic and negative charged property of carboxylic acid group on butylbenzoic acid, which repulsed the approach of both $\mathrm{C}-\mathrm{F}$ tails and $\mathrm{SO}_{3}$ groups. Considering that the HA/FA contained high hydrophilic and polar groups, the results of PFOS distribution on butylbenzoic acid modified surface agreed well with the experimental observation that HA/FA play an unimportant role on increasing PFOS adsorption on soil/sediment.
Based on the distribution structures and density profiles of PFOS on four surfaces, we concluded that PFOS adsorption benefited much more from the $\mathrm{SiO}_{2}$ surface and hexane/ butylbenzene modified surface rather than butylbenzoic modified surface. Then, the contribution of the soil/sediment components to PFOS adsorption followed the sequence: humin/kerogen $\geq$ inorganic component $>\mathrm{HA} / \mathrm{FA}$, which is consistent with our batch experiments (Fig. 3).

\subsection{Mean squared displacement (MSD)}

The average value of molecule displacement was calculated using MSD. The MSD of PFOS and $\mathrm{H}_{2} \mathrm{O}$ molecules on various surfaces during all NVT simulated time are shown in Appendix A Fig. S4. Generally, higher mobility of the molecules could lead to a larger slope in the MSD curve. A linearity (the slope converged to 1$)$ of the $\log (\mathrm{MSD})$ versus $\log ($ time) reflected that the equilibrium was achieved in our systems. The self-diffusion coefficients in Appendix A Table S4, calculated based on the Einstein relation, could be applied as index of the diffusion rate in the adsorption processes. 

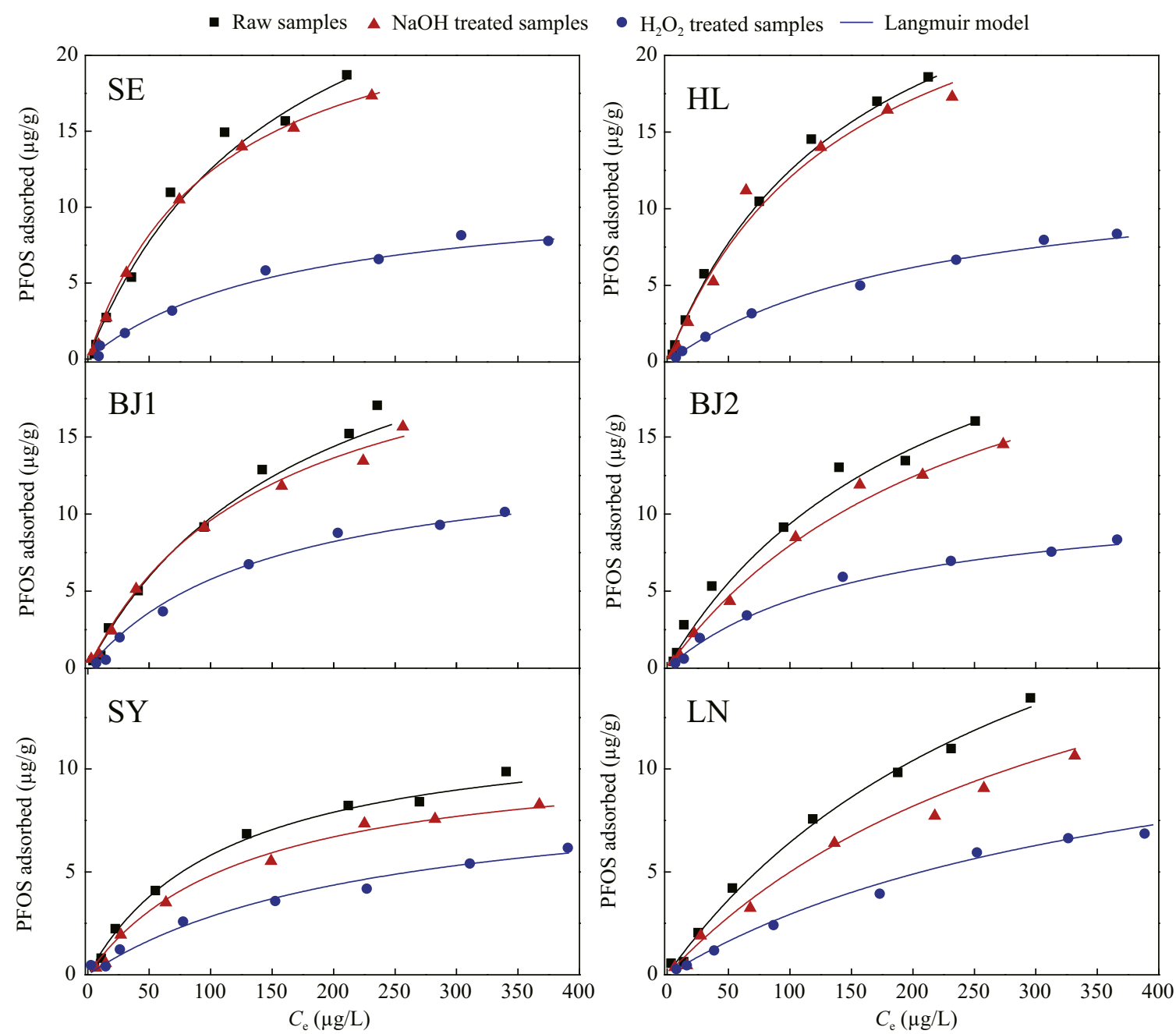

Fig. 2 - Adsorption isotherms of PFOS (perfluorooctane sulfonate) on soil/sediment samples at pH = 7, PFOS = 10-500 $\mu$ g/L, adsorbents $=15 \mathrm{~g} / \mathrm{L}$.

All diffusion coefficients on four surfaces were at the order of $10^{-6} \mathrm{~cm}^{2} / \mathrm{sec}$ (Appendix A Table S4), which was in agreement with previous reported data of benzene molecules

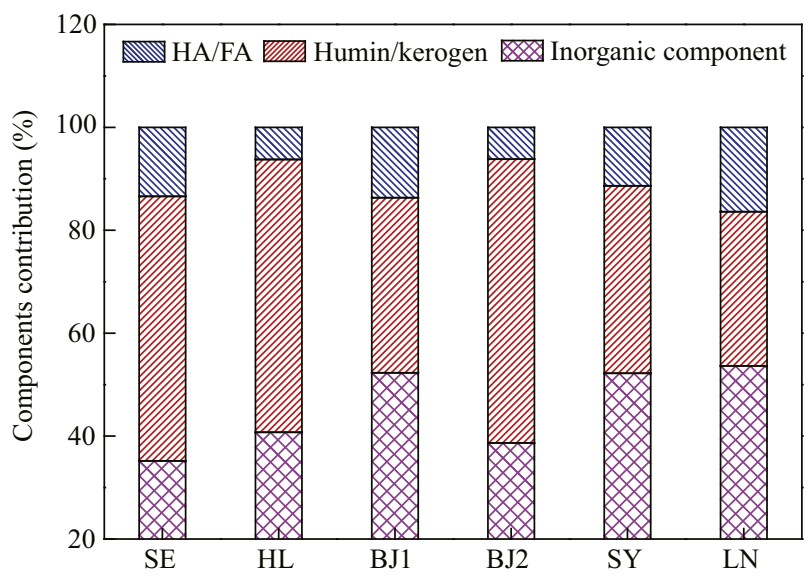

Fig. 3 - Contribution of different components (humic/fulvic acid (HA/FA), humin/kerogen, inorganic component) to PFOS adsorption on soil/sediment samples. diffusing in the interlayers of montmorillonite with organic materials (Zhao and Burns, 2012). Furthermore, the smallest diffusion coefficient $\left(3.1 \times 10^{-6} \mathrm{~cm}^{2} / \mathrm{sec}\right)$ of PFOS occurred on hexane modified (humin/kerogen) surface. This smallest diffusion coefficient was due to the phase transformation of PFOS from water phase to hexane phase (Zhao and Burns, 2012), indicating that PFOS owns the lowest mobility and higher affinity on humin/kerogen surface (Figs. 3 and 4).

\subsection{Radial distribution function}

To investigate the interactions of PFOS with their surrounding molecules, radial distribution function (RDF) was calculated to provide insight into the sorption of PFOS on four surfaces. In this study, the interaction was inferred using RDF among the groups of $\mathrm{F}-\mathrm{F}, \mathrm{F}-\mathrm{H}, \mathrm{SO}_{3}-\mathrm{H}$, and $\mathrm{SO}_{3}-\mathrm{SO}_{3}$ between PFOS and water molecules (Fig. 6).

As shown in Fig. 6a, no obvious peak of interaction between F-F atoms from different PFOS molecules was observed on $\mathrm{SiO}_{2}$ surface, hexane and butylbenzene modified surface. Conversely, two peaks at about 2.9 and $6.0 \AA$ were observed on butylbenzoic acid modified surface, suggesting 


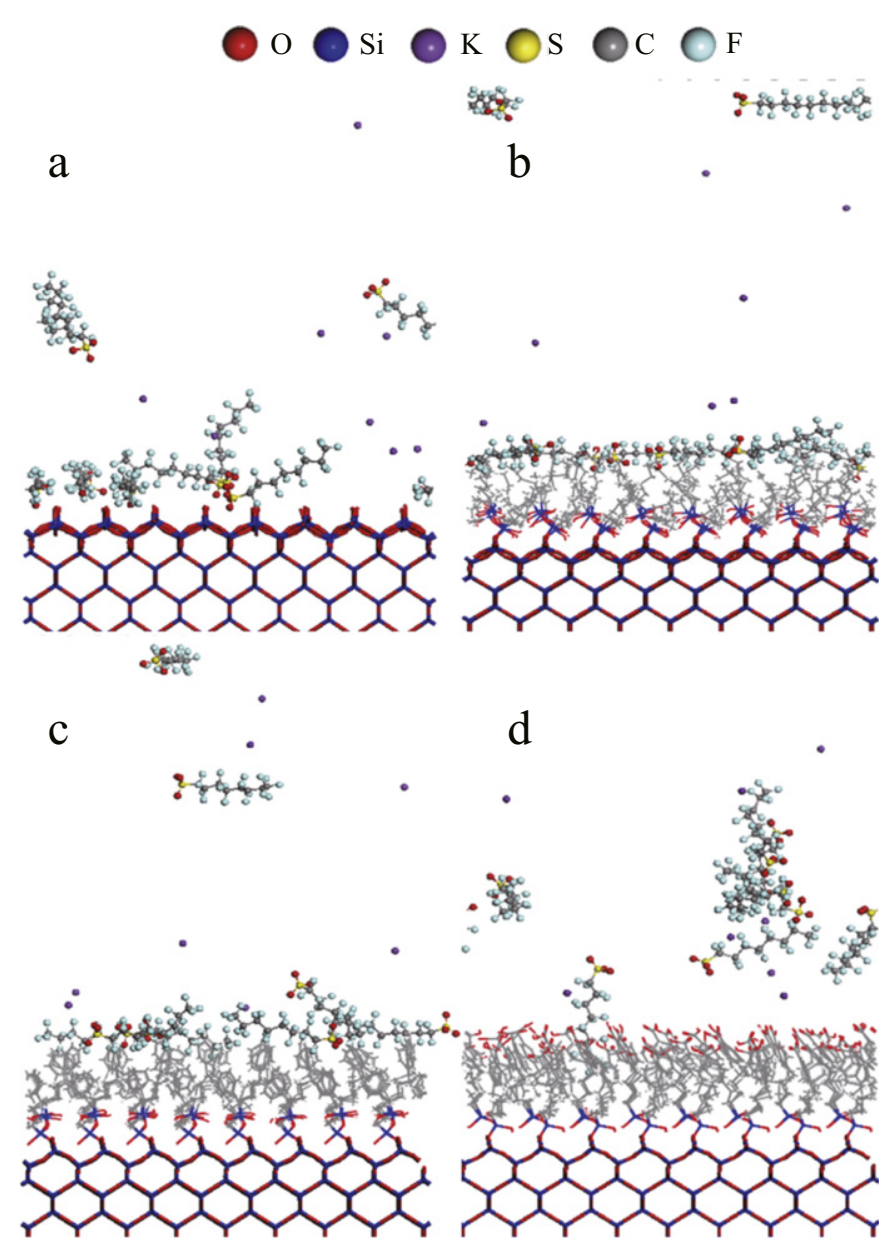

Fig. 4 - Snapshots of the simulated systems after equilibrium. (a) $\mathrm{SiO}_{2}$ surface, (b) hexane modified, (c) butylbenzene modified, (d) butylbenzoic acid modified $\mathrm{SiO}_{2}$ surface on side view. The water molecules were removed for visibility.

the strong F-F interaction force. Because of this F-F interaction, micelle was formed in the solution on butylbenzoic acid modified surface (Figs. $4 \mathrm{~d}$ and $6 \mathrm{a}$ ). Our results are in line with the finding that the perfluorinated surfactants are opt to associate with fluorous matrices (Chen et al., 2013).

For F-H interaction, three weak peaks at 1.9, 3.7, and $6.4 \AA$ (Fig. 6b) were observed, indicating weak interaction between PFOS molecules and water. The $1.9 \AA \mathrm{C}-\mathrm{F}$ distance is within the sum of van der Waals radii of fluorine (1.47 $\AA$ ) and hydrogen (1.20 ̊) (Howard et al., 1996), suggesting the formation of hydrogen bond.

Similarly, a strong peak at $1.7 \AA$ (Fig. 6c) for $\mathrm{SO}_{3}-\mathrm{H}$ interaction indicated the existence of hydrogen bond between $\mathrm{SO}_{3}$ group in PFOS molecules and water. In addition, the existence of $\mathrm{SO}_{3}-\mathrm{SO}_{3}$ interaction was evidenced by the broad peak around $5.0 \AA ̊$ (Fig. 6d).

\section{Conclusions}

Three major soil/sediment components including humin/ kerogen, HA/FA, and inorganic component were isolated and their contributions to PFOS adsorption were investigated using batch adsorption experiments and MD simulations. The order of the PFOS affinity on different surfaces follows humin/kerogen > inorganic component > HA/FA. Humin/kerogen dominated PFOS adsorption due to its aliphatic and nonpolar parts which facilitate phase transfer and hydrophobic

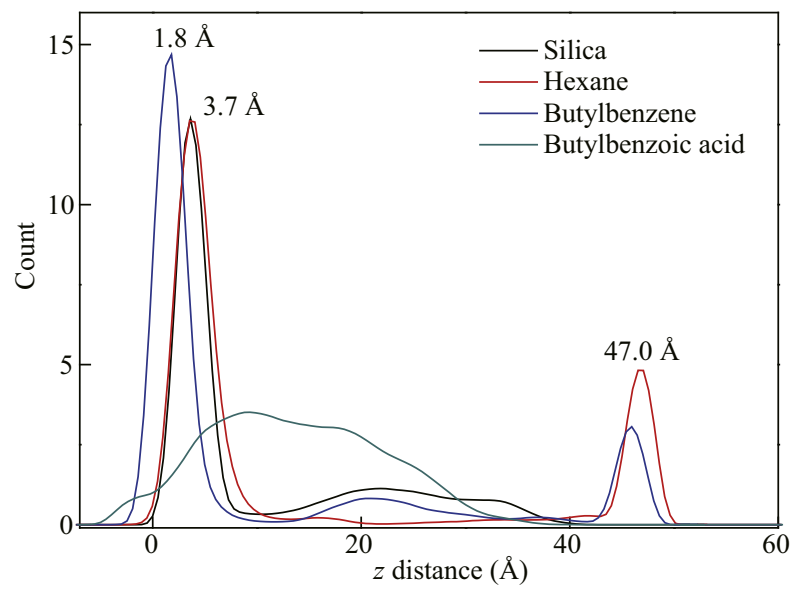

Fig. 5 - Density distributions of $\mathrm{PFOS}^{-}$on $\mathrm{SiO}_{2}$ and organic molecules modified $\mathrm{SiO}_{2}$ surfaces. 


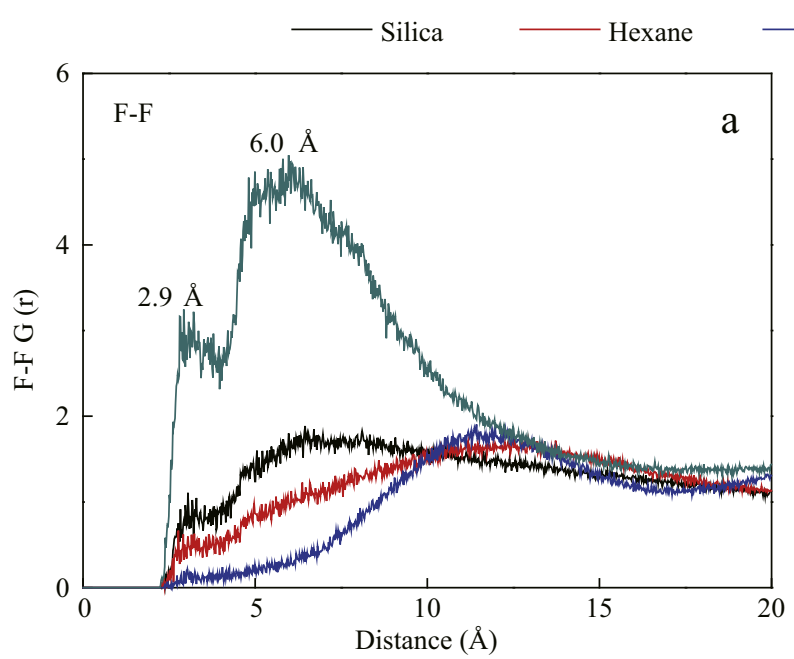

Butylbenzene $\quad$ Butylbenzoic acid
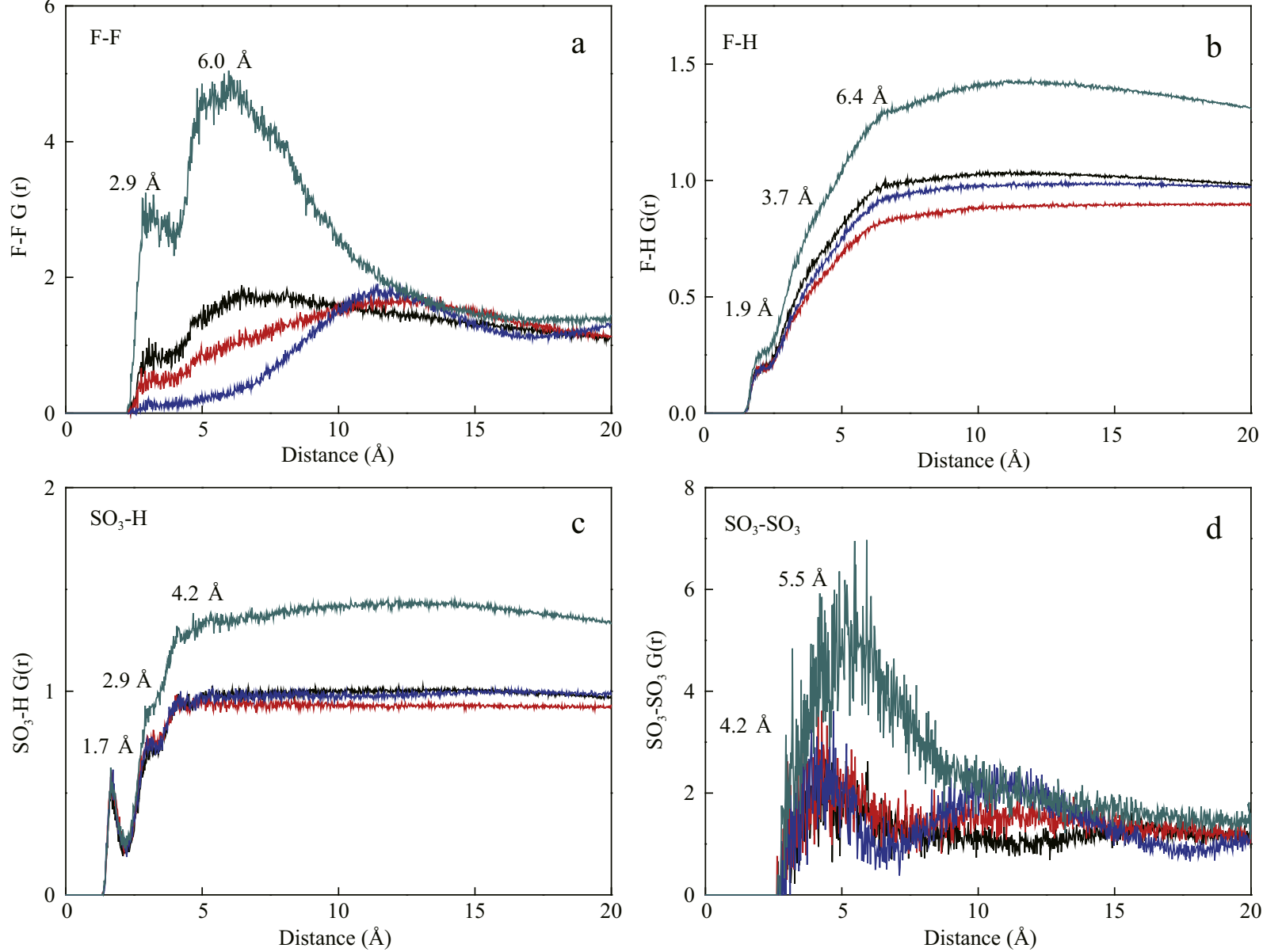

Fig. 6 - Radial distribution function (RDF) of interactions between $\mathrm{F}-\mathrm{F}$ atoms (a), $\mathrm{F}-\mathrm{H}$ atoms (b), $\mathrm{SO}_{3}-\mathrm{H}$ atoms (c), and $\mathrm{SO}_{3}-\mathrm{SO}_{3}$ (d) on silica surface, hexane-modified surface, butylbenzene-modified surface, and butylbenzoic acid-modified surface.

effect. Compared with humin/kerogen, HA/FA contributed much less to PFOS adsorption because its hydrophilic and polar features may increase the electrostatic repulsion and weaken the hydrophobic effect. The inorganic component also played an important role in the PFOS adsorption, possibly due to the formation of chemical bond with surface.

The results of the present study indicate that both organic substances (especially humin/kerogen) and inorganic component were attributed to PFOS adsorption on soil and sediment. Our findings imply that PFOS adsorption mechanisms are dependent on the composition and properties of soil and sediment. Thus, a comprehensive property of organic and inorganic components should be considered to quantify the fate and transport of PFOS in the environment.

\section{Acknowledgments}

This work was supported by the National Basic Research Program (973) of China (No. 2014CB441102), the Strategic Priority Research Program of the Chinese Academy of Sciences (No. XDB14020201), and Research Center for Eco-Environmental Sciences, Chinese Academy of Sciences (No. YSW2013A01). We also thank Dr. Wei Han for providing soil samples.

\section{Appendix A. Supplementary data}

Supplementary data associated with this article can be found in online version at http://dx.doi.org/10.1016/j.jes.2014.11.001.

\section{R E F E R E N C E S}

Aquino, A.J.A., Tunega, D., Pašalić, H., Schaumann, G.E., Haberhauer, G., Gerzabek, M.H., et al., 2011. Molecular dynamics simulations of water molecule-bridges in polar domains of humic acids. Environ. Sci. Technol. 45 (19), 8411-8419.

Beach, S.A., Newsted, J.L., Coady, K., Giesy, J.P., 2006. Ecotoxicological evaluation of perfluorooctanesulfonate (PFOS). Rev. Environ. Contam. Toxicol. 186, 133-174.

Betts, K.S., 2007. Perfluoroalkyl acids: what is the evidence telling us? Environ. Health Perspect. 115 (5), 250-266.

Betts, K.S., 2008. Not immune to PFOS effects? Environ. Health Perspect. 116 (7), A290.

Chen, H., Zhang, C., Yu, Y.X., Han, J.B., 2012. Sorption of perfluorooctane sulfonate (PFOS) on marine sediments. Mar. Pollut. Bull. 64 (5), 902-906.

Chen, L.D., Lai, C.Z., Granda, L.P., Fierke, M.A., Mandal, D., Stein, A., et al., 2013. Fluorous membrane ion-selective electrodes for 
perfluorinated surfactants: trace level detection and in-situ monitoring of adsorption. Anal. Chem. 85 (15), 7471-7477.

Giannakopoulos, E., Stathi, P., Dimos, K., Gournis, D., Sanakis, Y., Deligiannakis, Y., 2006. Adsorption and radical stabilization of humic-acid analogues and $\mathrm{Pb}^{2+}$ on restricted phyllomorphous clay. Langmuir 22 (16), 6863-6873.

Gump, B.B., Wu, Q., Dumas, A.K., Kannan, K., 2011. Perfluorochemical (PFC) exposure in children: associations with impaired response inhibition. Environ. Sci. Technol. 45 (19), 8151-8159.

Han, W., Luo, L., Zhang, S.Z., 2013. Adsorption of tetrabromobisphenol A on soils: contribution of soil components and influence of soil properties. Colloids Surf. A Physicochem. Eng. Asp. 428, 60-64.

He, G.Z., Pan, G., Zhang, M.Y., 2013. Assembling structures and dynamics properties of perfluorooctane sulfonate (PFOS) at water-titanium oxide interfaces. J. Colloid Interface Sci. 405, 189-194.

Higgins, C.P., Luthy, R.G., 2006. Sorption of perfluorinated surfactants on sediments. Environ. Sci. Technol. 40 (23), 7251-7256.

Higgins, C.P., Luthy, R.G., 2007. Modeling sorption of anionic surfactants onto sediment materials: an a priori approach for perfluoroalkyl surfactants and linear alkylbenzene sulfonates. Environ. Sci. Technol. 41 (9), 3254-3261.

Howard, J.A.K., Hoy, V.J., O’Hagan, D., Smith, G.T., 1996. How good is fluorine as a hydrogen bond acceptor? Tetrahedron 52 (38), 12613-12622.

Hu, Y.F., Lü, W.J., Shang, Y.Z., Liu, H.L., Wang, H.L., Suh, S.H., 2013. DMSO transport across water/hexane interface by molecular dynamics simulation. Ind. Eng. Chem. Res. 52 (19), 6550-6558.

Johnson, R.L., Anschutz, A.J., Smolen, J.M., Simcik, M.F., Penn, R.L., 2007. The adsorption of perfluorooctane sulfonate onto sand, clay, and iron oxide surfaces. J. Chem. Eng. Data 52 (4), 1165-1170.

Kang, S.H., Xing, B.S., 2005. Phenanthrene sorption to sequentially extracted soil humic acids and humins. Environ. Sci. Technol. 39 (1), 134-140

Kannan, K., Yun, S.H., Rudd, R.J., Behr, M., 2010. High concentrations of persistent organic pollutants including PCBs, DDT, PBDEs and PFOS in little brown bats with white-nose syndrome in New York, USA. Chemosphere 80 (6), 613-618.

Liu, P., Zhu, D.Q., Zhang, H., Shi, X., Sun, H.Y., Dang, F., 2008. Sorption of polar and nonpolar aromatic compounds to four surface soils of eastern China. Environ. Pollut. 156 (3), 1053-1060.

Luebker, D.J., York, R.G., Hansen, K.J., Moore, J.A., Butenhoff, J.L., 2005. Neonatal mortality from in utero exposure to perfluorooctanesulfonate (PFOS) in Sprague-Dawley rats: dose-response, and biochemical and pharamacokinetic parameters. Toxicology 215 (1-2), 149-169.

Luo, L., Zhang, S.Z., Ma, Y.B., 2008. Evaluation of impacts of soil fractions on phenanthrene sorption. Chemosphere 72 (6), 891-896.

Mikutta, R., Kleber, M., Kaiser, K., Jahn, R., 2005. Review: organic matter removal from soils using hydrogen peroxide, sodium hypochlorite, and disodium peroxodisulfate. Soil Sci. Soc. Am. J. 69 (1), 120-135.
Nakayama, S.F., Strynar, M.J., Reiner, J.L., Delinsky, A.D., Lindstrom, A.B., 2010. Determination of perfluorinated compounds in the upper Mississippi river basin. Environ. Sci. Technol. 44 (11), 4103-4109.

Nelson, J.W., Hatch, E.E., Webster, T.F., 2010. Exposure to polyfluoroalkyl chemicals and cholesterol, body weight, and insulin resistance in the general US population. Environ. Health Perspect. 118 (2), 197-202.

Ochoa-Herrera, V., Sierra-Alvarez, R., 2008. Removal of perfluorinated surfactants by sorption onto granular activated carbon, zeolite and sludge. Chemosphere 72 (10), 1588-1593.

Plimpton, S., 1995. Fast parallel algorithms for short-range molecular dynamics. J. Comput. Phys. 117 (1), 1-19.

Shi, X., Ji, L.L., Zhu, D.Q., 2010. Investigating roles of organic and inorganic soil components in sorption of polar and nonpolar aromatic compounds. Environ. Pollut. 158 (1), 319-324.

Sun, H., 1998. COMPASS: an ab initio force-field optimized for condensed-phase applications overview with details on alkane and benzene compounds. J. Phys. Chem. B 102 (38), 7338-7364.

Sun, Q., Xie, H.B., Chen, J.W., Li, X.H., Wang, Z., Sheng, L.X., 2013. Molecular dynamics simulations on the interactions of low molecular weight natural organic acids with $\mathrm{C}_{60}$. Chemosphere 92 (4), 429-434.

Tang, C.Y., Shiang Fu, Q., Gao, D., Criddle, C.S., Leckie, J.O., 2010. Effect of solution chemistry on the adsorption of perfluorooctane sulfonate onto mineral surfaces. Water Res. 44 (8), 2654-2662.

Verlet, L., 1967. Computer "Experiments" on classical fluids. I. Thermodynamical properties of Lennard-Jones molecules. Phys. Rev. 159 (1), 98-103.

Wang, X.L., Guo, X.Y., Yang, Y., Tao, S., Xing, B.S., 2011. Sorption mechanisms of phenanthrene, lindane, and atrazine with various humic acid fractions from a single soil sample. Environ. Sci. Technol. 45 (6), 2124-2130.

You, C., Jia, C.X., Pan, G., 2010. Effect of salinity and sediment characteristics on the sorption and desorption of perfluorooctane sulfonate at sediment-water interface. Environ. Pollut. 158 (5), 1343-1347.

Zhang, K.L., Huang, J., Yu, G., Zhang, Q.W., Deng, S.B., Wang, B., 2013. Destruction of perfluorooctane sulfonate (PFOS) and perfluorooctanoic acid (PFOA) by ball milling. Environ. Sci. Technol. 47 (12), 6471-6477.

Zhao, Q., Burns, S.E., 2012. Molecular dynamics simulation of secondary sorption behavior of montmorillonite modified by single chain quaternary ammonium cations. Environ. Sci. Technol. 46 (7), 3999-4007.

Zhao, Q., Burns, S.E., 2013. Modeling sorption and diffusion of organic sorbate in hexadecyltrimethylammonium-modified clay nanopores-a molecular dynamics simulation study. Environ. Sci. Technol. 47 (6), 2769-2776.

Zhao, T.T., Xu, G.Y., Yuan, S.L., Chen, Y.J., Yan, H., 2010. Molecular dynamics study of alkyl benzene sulfonate at air/water interface: effect of inorganic salts. J. Phys. Chem. B 114 (15), 5025-5033. 